\title{
The fourth pillar
}

\author{
Despite some setbacks in the clinic, immunotherapy has made notable progress toward becoming an \\ additional therapeutic option against cancer
}

\author{
Philip Hunter
}

$\mathrm{D}$ espite decades of research, the triumvirate of surgery, radiotherapy, and chemotherapy-or "slash, burn, and poison"-still remain as the three main pillars of cancer therapy. At one time, apoptosis, programmed cell suicide, instilled great hopes of future therapies that could coerce cancer cells to commit seppuku, but after more than 20 years of research no such approach has made it to the clinic. At another time, RNA interference held great promise to become a fourth pillar of anticancer drugs that were more specific and less toxic than chemotherapy, but it proved much harder than anticipated to deliver these fragile molecules to target sites and persuade cells to take them up. Rational drug design to create molecules that target cancer-specific proteins has also yielded little progress in terms of new and more efficient drugs.

cc

... immunotherapy has

shown mixed results with great variations in efficacy between patients and cancer types"

It was therefore natural that the cancer research field would be skeptical over claims that immunotherapy could become the fourth pillar of treatment even after the first cancer vaccines were approved around 2010. Indeed, the setbacks of some clinical trials reinforced that skepticism. In July 2017, AstraZeneca announced disappointing results for its global Phase III MYSTIC trial of the drug durvalumab either on its own or in combination with standard-of-care chemotherapy in previously untreated patients with metastatic non-small cell lung cancer. In both cases, the treatment failed to improve progression-free survival over the standard care in patients whose tumors express the protein PD-L1 (programmed death ligand). PD-L1 binds to the PD-1 receptor on activated $\mathrm{T}$ cells and thereby inhibits or at least modulates their ability to attack cancer cells.

\section{Checkpoint inhibitors}

PD-1 is also a so-called checkpoint protein because it enables the immune system to control the action of $\mathrm{T}$ cells by either turning activation signals up or down. A natural target for immunotherapy is therefore the development of checkpoint inhibitors that either block checkpoint proteins such as PD-1 on $\mathrm{T}$ cells or proteins like PD-L1 on tumor cells. Durvalumab, also known by the trademarked name Imfinzi, blocks the interaction between PD-L1 and PD-1.

However, the failure of clinical trials just highlights the need for further research to understand the complex interaction between the immune system and tumors rather than being a significant setback for immunotherapy. A key point is that immunotherapy has shown mixed results with great variations in efficacy between patients and cancer types. This dichotomy is well illustrated by the case of durvalumab itself, because just a few days after the report of disappointing results, the drug was granted special breakthrough status by the FDA for treating patients with unresectable non-small cell lung cancer. This was based on results showing efficacy in patients who had been initially treated by chemoradiation therapy and had remained in remission but at risk of the disease returning. Until now, the only option for these patients was active monitoring, followed by further chemotherapy or radiotherapy if the cancer returns. As the FDA commented, this was the first immunooncology medicine to show clinically significant benefits at an earlier stage before the cancer had metastasized.

The results from durvalumab trials against early-stage lung cancer are in line with findings from animal studies, according to Douglas McNeel, Director of Solid Tumor Immunology Research at the Wisconsin Institutes for Medical Research in Madison, USA. "In animal models we know that antitumor vaccines, for example, work best in the setting of minimal tumor burden", he commented. "This will likely be the case in clinical trials. To get there we need to understand short-term markers of clinical effect and benefit to enable clinical trials to be more efficiently performed on patients with early stages of disease".

"There is additional scope for using checkpoint inhibitors in combination with other therapies, including vaccines, to stimulate the immune response"

There is already cause for optimism, McNeel reckoned. "Recent progress has been fantastic", he said. "Recall that there were many decades of clinical research with single agent therapies, notably vaccines, adoptive immunotherapies and cytokines, that made very slow progress and led to lots 
of skepticism for the whole field. Perhaps the greatest impact recently has been identifying mechanisms of tumor resistance to immune treatment, findings that have enabled the evaluation of T-cell checkpoint therapies. These agents, used alone, have made major impacts for several cancers".

\section{Personalized anti-cancer vaccines}

There is additional scope for using checkpoint inhibitors in combination with other therapies, including vaccines, to stimulate the immune response. This could work in cases where checkpoint inhibitors alone have failed, as in that recent AstraZeneca trial. Unlike vaccines for infectious diseases, solid cancer vaccines are therapeutic and can only work after a tumor has already developed. Most commonly, this involves extraction of antigen proteins from cancer cells and injecting those into the patient in the hope of stimulating the immune system to target more $\mathrm{T}$ cells at the tumor.

One of the first cancer vaccines with proven efficacy was sipuleucel-T, trademarked Provenge, approved by the FDA in April 2010 for use in men with metastatic prostate cancer that could not be removed surgically. In this case, the precise mechanisms have still not been elucidated, but it appears to stimulate $\mathrm{T}$ cells to kill tumor cells that express the protein PAP. The vaccine was prepared by culturing PAP with another protein called granulocytemacrophage colony-stimulating factor (GM-CSF), which is known to stimulate the immune system and enhance presentation of antigens. It seems that this makes the antigens more visible to the T-cell compartments where initial immune recognition takes place.

Sipuleucel-T was an early example of a personalized cancer immunotherapy, as it uses antigen-presenting dendritic immune cells isolated from the patient's blood. The key starting point is that cancer only arises in the first place from a failure of immune recognition, or else it would not be there. "All immune therapies attempt in some way to correct that failure", explained Ton Schumacher, Group Leader at the Netherlands Cancer Institute in Amsterdam. "There are cases where the tumors do have antigens that could be seen by the $\mathrm{T}$ cell compartments, but the $\mathrm{T}$ cell priming and activation process is sub-optimal. That is a perfect scenario for a personalized vaccine to induce immune response and then we would expect synergy with molecules like anti-PD1 which maximize the activity of the $\mathrm{T}$ cells you've induced".

\section{"One attraction of the RNA platform is the relative speed of drug development and the potential to reduce costs, both of which are essential for personalized therapies"}

This kind of combination therapy to induce or amplify immune recognition and action at the same time is likely to gain wider acceptance, according to McNeel. "We have recently found that DNA vaccination with PD-1 blockade can elicit anti-tumor immune responses leading to a reduction in tumor volume in patients with advanced prostate cancer", he said [1]. "So T cells are augmented and activated with vaccination, and PD-1 blockade at the time of vaccination allows them to function better. [...] I think the future will include combinations of multiple agents targeting immunosuppressive mechanisms within the tumor environment, vaccines or other therapies augmenting tumor-specific lymphocytes, and T-cell checkpoint inhibitors and other T-cell activating agents that maintain the activity of these tumor-specific lymphocytes".

\section{CAR T cells}

Apart from vaccination against specific antigens and blockading of immune checkpoints, another promising approach involves genetically modifying $\mathrm{T}$ cells so that they can better recognize tumor cells. "There for every patient you have to harvest the T cells, genetically modify them in vitro and then reinfuse them", Schumacher explained the so-called Chimeric Antigen Receptor T cells or CAR T cells. "So this again is an individual product designed for each patient separately". Like other personalized therapies, this is expensive, especially at first, and therefore must show sufficient potential to justify the upfront costs and ongoing investment to make it more affordable for a greater number of patients. "Early trials have showed such profound clinical activity with a significant fraction of patients showing durable responses that people in the field think it's worth the effort”, Schumacher said. "The clinical signal is so strong that we are likely to see approvals for modified T cells".

Three biotech companies, Novartis, Kite Pharma, and Juno Therapeutics, have been preparing applications for approval of T-cell therapies for aggressive blood cancers. In June 2017, Novartis announced interim results from its Phase II JULIET study in 51 adult patients with relapsed or refractory diffuse large B-cell lymphoma (DLBCL): 37\% of patients showed complete responses, or no sign of disease (https://www.novartis.com/ news/media-releases/novartis-interim-resultsglobal-pivotal-ctl019-trial-show-durable-comp lete). This is considered a good result for relapsed patients and puts the company on track to obtain FDA approval to market the first CAR T-cell therapy. It followed a unanimous vote by the FDA's Oncologic Drugs Advisory Committee (ODAC) to recommend approval of this drug, called CTL019.

Kite Pharma, with which Schumacher is involved, has also developed $\mathrm{T}$ cells modified to express CAR proteins, as well as an alternative approach called $\mathrm{T}$-cell receptor (TCR). The latter differs in isolating endogenous proteins from the patient that are known to be specific to a cancer rather than designing novel chimeric proteins from scratch. These are then engineered into $\mathrm{T}$ cells that are again collected from the patient. The TCR approach can be applied to solid as well as blood cancers, according to the company. Kite recently filed applications with the EMA (European Medical Agency) for its version of the CAR T-cell therapy, called Axi-cel.

\section{RNA vaccines}

There has also been progress with novel cancer vaccines against tumors that are resistant to immunotherapy. Here too, the therapies are patient-specific, and one of the challenges lies in making vaccine development practical and affordable. RNA vaccines that encode mutant or endogenous antigens associated with tumors have emerged as promising candidates after a phase 1 trial showed strong effector and memory T-cell responses and potent rejection of progressive tumors [2]. These RNA-lipoplexes could overcome various technical challenges associated with penetration of dendritic antigenpresenting cells that transmit antigen to $\mathrm{T}$ cells.

"Our findings show that individual cancer mutations are excellent cancer 
vaccine targets”, commented Özlem Türeci, one of the study's authors at the Immunology Cluster of Excellence at Johannes Gutenberg-Universität in Mainz, Germany, who is also co-founder and CEO of Ganymed Pharmaceuticals AG, a biotech company developing antibody drugs. "Our vaccine was capable of inducing an immune response against the cancer in each and every patient, also in patients with relatively low numbers of mutations", she said. "We also expect that our vaccine will work in cancer types with low mutation rate, which is the hurdle for checkpoint inhibitors that also rely on mutation-specific T cells".

Nonetheless, checkpoint inhibitors can amplify T-cell response to tumors. Ganymed Pharmaceuticals is therefore working with Roche subsidiary Genentech to combine its vaccine with the latter's checkpoint inhibitor. "The rationale here is that checkpoint inhibitors unleash those neo-epitope-specific $\mathrm{T}$ cells, which are already existent”, Türeci elucidated. "The vaccine, however, can generate novel $\mathrm{T}$ cells against mutations which have so far not been immune recognized by the patient". Türeci anticipates the first clinical approval for a cancer vaccine to come around 2022. "As in any drug development, our vaccine has to pass clinical trial Phases 1, 2 and 3 and prove superiority in randomized controlled comparison with non-personalized state-of-art treatments", she said. "Along these phases we will for example assess a new, systemically administrable formulation, combine with other compounds and established treatment regimens, and further improve manufacturing in terms of speed and costs". She also anticipates that the vaccine could help to treat patients with recurrent cancers. "Since the tumor changes with each recurrence, we can design a new personalized vaccine each time”, Türeci explained.

One attraction of the RNA platform is the relative speed of drug development and the potential to reduce costs, both of which are essential for personalized therapies. "One of the big challenges is to develop a vaccine which really addresses the target antigens in time”, said Ugur Sahin, Managing Director and Co-Founder of TRON (Translational Oncology at the University Medical Center of the Johannes Gutenberg University Mainz) and Türeci's husband. "One advantage of our synthetic RNA vaccine is that it can be prepared much faster than before. This means getting the information from the tumor and making vaccine is now feasible". The development platform has been designed for universal application so it can be used in principle to develop vaccines for any tumor. "We use a modular system where, depending on the antigen constellation of the patient, we just change the vaccine antigens and then use the same vaccine system”, Sahin explained.

\section{Prevention versus cure}

In any case, costs need to be balanced against clinical benefit. As Schumacher pointed out, surgery is the ultimate personalized therapy and nobody has ever suggested abandoning it to save money. Equally though, nobody would deny that the ideal treatment for any disease is preventative rather than therapeutic, which is why there is also interest in the idea of an upfront vaccine encouraging the immune system to patrol for antigens indicative of cancer before it occurs. Such a preventative vaccine could not be derived from mutagenic cancer antigens because as Schumacher explained, these tend to be highly specific to a given cancer. "The overlap in mutations between any pair of tumors is very small, which makes it difficult to determine which ones will appear in a tumor later in life to design a vaccine", he commented.

(c

... there is ongoing work to develop vaccines based on non-mutant antigens that are expressed differently on tumors compared to normal tissue as a result of epigenetic changes. .."

However, there is ongoing work to develop vaccines based on non-mutant antigens that are expressed differently on tumors compared to normal tissue as a result of epigenetic changes associated with the tumor microenvironment. One such antigen called MUC1 was first identified as playing a role in cancer 26 years ago [3]. It provided the basis for recent development of a vaccine by Olivera Finn and colleagues at the University of Pittsburgh School of Medicine in the USA. "I am very optimistic that the preventative cancer vaccines will work using the shared tumor-associated antigens that are also disease associated antigens",
Finn said. "The reason is that these vaccines boost existing immune memory, which is easier to do in an adult patient at risk for cancer than prime new immune responses to unique mutations presented by the cancer. [...]. We published a study in 2013 [4] on testing immunogenicity and safety of the MUC1 vaccine in individuals with a history of advanced polyps, precursors to colon cancer. We showed it to be immunogenic and safe and currently we are running a multi-center, placebo controlled efficacy trial, funded by the Division of Cancer Prevention of the National Cancer Institute".

There are several reasons for choosing MUC1 as an antigen for a preventative cancer vaccine, according to Finn: "MUC1 is over-expressed and hypo-glycosylated in tumors compared to normal epithelial cells. In normal cells, it is present only on the apical surface facing the internal luminal face of a duct, which is sort of outside of the body, so that it is sequestered from the immune system. Also, MUC1 is abnormally trafficked to be expressed all over the tumor cell surface. All of this generates improper, or lack of, control of MUC1 synthesis and posttranslational modifications”.

There are also some other factors that change MUC1 expression, including local inflammation resulting in production of proinflammatory cytokines such as TNF and IL-6 that promote increased MUC1 transcription. "Too much MUC1 protein leads to its reduced glycosylation”, Finn explained. "So at the site of inflammation, especially chronic inflammation, you find overexpression of the hypoglycosylated form of MUC1, the form that is also expressed on tumor cells".

This leads to the interesting possibility that MUC1 could also be a candidate antigen for vaccines against infectious and autoimmune disease characterized by chronic inflammation. "While studying MUC1 and another tumor antigen that we discovered, cyclin B1, we made an important observation that many tumor-associated antigens (TAA) described by their abnormal expression on cancer cells are also abnormally expressed in other acute or chronic inflammatory conditions and are therefore more appropriately defined as disease associated antigens (DAA)", Finn commented. "These include bacterial infections such as Mumps, viral infections such as chicken pox, and chronic inflammations such as inflammatory bowel disease (IBD)". 


\section{Not quite there yet}

However, the main focus for Finn is on cancer and one of the challenges is establishing efficacy for a preventative therapy against a disease that might not occur anyway. Trials would need to be very long term and might never be conclusive if such vaccines merely reduced the risk slightly. Partly for this reason, preventative vaccines may be confined at first to people deemed at risk, in the event that safety has already been proven. "Preventative vaccines alone should work very well and should be enough, perhaps with a yearly booster, in people at risk but no evidence of disease", Finn said. "However, we have shown that some premalignant lesions already establish an immunosuppressive microenvironment, so for those at high risk for progression, there might need to be combinations of vaccines with other immune modulators, including checkpoint inhibitors".

It is still early days for most forms of immunotherapy and the case for embracing them as that elusive fourth pillar has yet to be proven, despite the growing optimism. Perhaps the key mark of progress is that there is now a much better understanding of why some patients and tumors show a strong response while others show little or even none at all. "We are getting to know first which patients to select and secondly which treatments to give”, Schumacher commented.

\section{"Perhaps the key mark of progress is that there is now a much better understanding of why some patients and tumors show a strong response while others show little or even none at all"}

The one tangible marker of progress is the drug Keytruda, or pembrolizumab, granted approval under the FDA's Accelerated Approval pathway for drugs treating serious conditions where there is no current treatment. This can now be given to patients with solid tumors after prior treatment with no satisfactory alternative treatment options left. "That was the first FDA approval for treating cancers characterized by a specific genetic aberration, rather than location in the body", Schumacher said. It targets cancers with a microsatellite instability high, which is a form of DNA damage associated with some solid cancers, especially colorectal. But even Keytruda requires further study to verify that the anticipated clinical benefits occur. That is the stage immunotherapy is at, progressing beyond early hype but not quite there to become a reliable therapeutic option for a wide range of cancers.

\section{References}

1. McNeel DG, Eickhoff JC, Jeraj R, Staab MJ, Straus J, Rekoske B, Liu G (2016) DNA vaccine with pembrolizumab elicits anti-tumor responses in patients with metastatic, castration-resistant prostate cancer (MCRPC). National meeting of the Society for Immunotherapy of Cancer, Washington, DC, USA, Abstract 369

2. Kranz LM, Diken M, Haas $H$, Kreiter $S$, Loquai C, Reuter KC, Meng M, Fritz D, Vascotto F, Hefesha $\mathrm{H}$ et al (2016) Systematic RNA delivery to dendritic cells exploits antiviral defence for cancer immunotherapy. Nature 534: $396-401$

3. Jerome KR, Barnd DL, Bendt KM, Boyer CM, Taylor-Papadimitrou J, McKenzie IFC, Bast RC Jr, Finn OJ (1991) Cytotoxic T-lymphocytes derived from patients with breast adenocarcinoma recognize an epitope present on the protein core of a mucin molecule preferentially expressed by malignant cells. Cancer Res 51: $2908-2916$

4. Kimura T, McKolanis JR, Dzubinski LA, Islam K, Potter DM, Salazar AM, Schoen RE, Finn O] (2013) MIC1 vaccine for individuals with advanced adenoma of the colon: a cancer immunoprevention feasibility study. Cancer

Preu Res 6: 18-26 